\title{
Automated Nanofiber Diameter Measurement in SEM Images Using a Robust Image Analysis Method
}

\author{
Ertan Öznergiz, ${ }^{1}$ Yasar Emre Kiyak, ${ }^{2}$ Mustafa E. Kamasak, ${ }^{3}$ and Isa Yildirim ${ }^{4}$ \\ ${ }^{1}$ Department of Marine Engineering Operations, Yildiz Technical University, 34349 Istanbul, Turkey \\ ${ }^{2}$ College of Textiles, North Carolina State University, Raleigh, NC 27606, USA \\ ${ }^{3}$ Faculty of Computer and Informatics, Istanbul Technical University, 34469 Istanbul, Turkey \\ ${ }^{4}$ Faculty of Electrical and Electronics Engineering, Istanbul Technical University, 34469 Istanbul, Turkey \\ Correspondence should be addressed to Ertan Öznergiz; oznergiz@yildiz.edu.tr
}

Received 24 June 2014; Accepted 16 October 2014; Published 6 November 2014

Academic Editor: Shuangxi Xing

Copyright (C) 2014 Ertan Öznergiz et al. This is an open access article distributed under the Creative Commons Attribution License, which permits unrestricted use, distribution, and reproduction in any medium, provided the original work is properly cited.

\begin{abstract}
Due to the high surface area, porosity, and rigidity, applications of nanofibers and nanosurfaces have developed in recent years. Nanofibers and nanosurfaces are typically produced by electrospinning method. In the production process, determination of average fiber diameter is crucial for quality assessment. Average fiber diameter is determined by manually measuring the diameters of randomly selected fibers on scanning electron microscopy (SEM) images. However, as the number of the images increases, manual fiber diameter determination becomes a tedious and time consuming task as well as being sensitive to human errors. Therefore, an automated fiber diameter measurement system is desired. In the literature, this task is achieved by using image analysis algorithms. Typically, these methods first isolate each fiber in the image and measure the diameter of each isolated fiber. Fiber isolation is an error-prone process. In this study, automated calculation of nanofiber diameter is achieved without fiber isolation using image processing and analysis algorithms. Performance of the proposed method was tested on real data. The effectiveness of the proposed method is shown by comparing automatically and manually measured nanofiber diameter values.
\end{abstract}

\section{Introduction}

Nanotechnology has recently become the most popular area of scientific and technological activities. Nanofibers have extremely high specific surface area due to their small diameters. Additionally, nanofiber mats can be highly porous with excellent pore interconnection. One-gram fiber can cover a surface up to $1000 \mathrm{~m}^{2}$ depending on the polymer thickness. These unique characteristics make nanofibers an excellent choice for advanced applications such as filtration, agrotextiles, and medical applications. Electrospinning has been the most effective technique for nanofiber production processes. This process technology ensures the efficient use of materials and provides a very high quality filtration. The following items are used in the electrospinning process: (1) a polymer solution, (2) a feeder that has a low feed rate which is used to transfer the polymer solution to the charging unit, (3) a conductive charging unit that is used to charge the polymer solution, (4) a high voltage power supply to charge the polymer passing through the charging unit, and (5) a grounded connector where the charged polymer is transferred due to the potential difference between charging unit and connector.

Automatic calculation of the fiber diameters is a relatively new research topic. There are few studies in the literature on automated fiber feature analysis. In these studies, fibers were first isolated by using image processing methods. After the isolation process, the direction $[1,2]$, diameter [3-6], pore size [7], uniformity [8], and other structural features [9] of the isolated fibers were investigated. Performance of these studies was tested on both synthetic and real data. Diameters of the real data nanofibers were measured manually for comparison. Performance of these methods may be adversely affected by the errors in the fiber isolation process. In this study, a novel image analysis method is developed to automate fiber diameter calculation without the need of fiber isolation stage.

The paper is organized as follows. In Section 2, the proposed method is described. The results of the proposed 


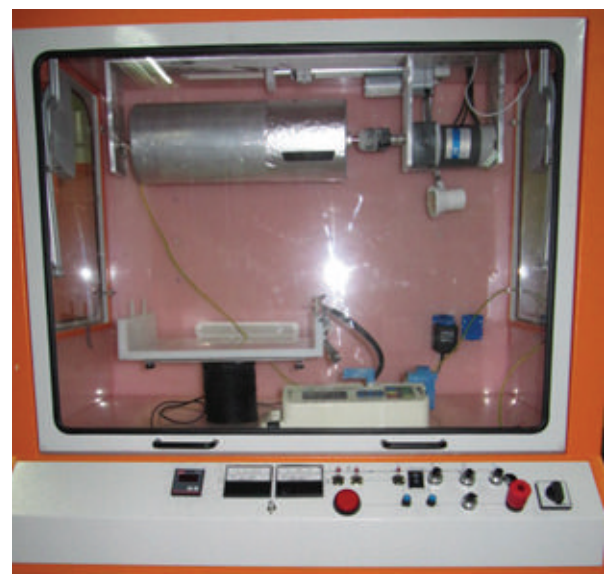

FIGURE 1: The MEP used for nanofiber production by electrospinning [1].

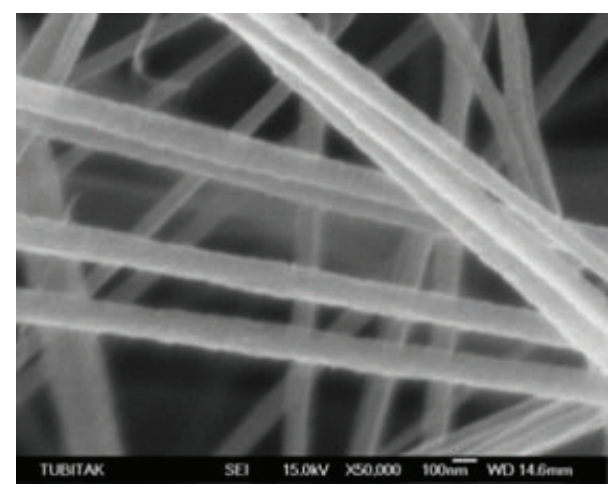

(a)

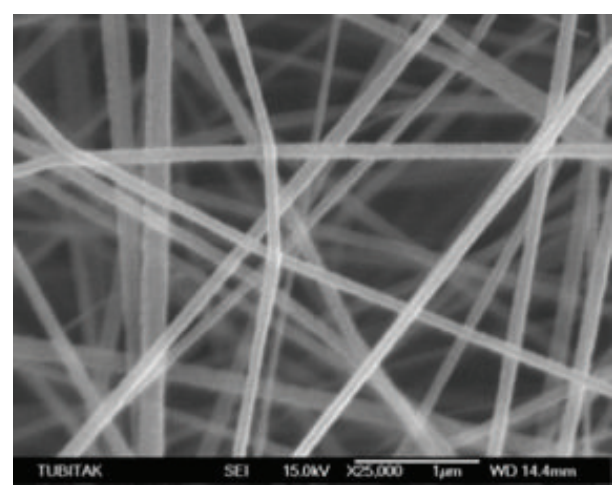

(b)

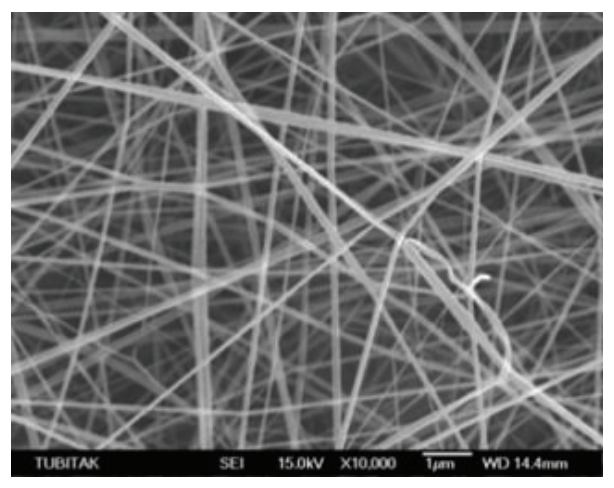

(c)

FIGURE 2: Sample SEM images at different resolutions of PAN/DMF ( $8 \%$ concentration) nanofiber mats.

method are demonstrated in Section 3. The paper is concluded in Section 4.

\section{SEM Image Analysis Method}

In this paper, the Multifunctional Electrospinning Platform (MEP) shown in Figure 1 is used in the production process [10]. The nanofibers examined in this study are produced using PAN/DMF, PU, PVA, PEO-PCL3 solutions of different concentrations, $12 \mathrm{kV}$ voltage, $14 \mathrm{~cm}$ needle to collector distance, and $1.2 \mathrm{~mL} / \mathrm{h}$ flow rate. SEM images at different resolutions of PAN/DMF ( $8 \%$ concentration) nanofiber mats are shown in Figure 2. The image analysis algorithm that is designed for automated nanofiber diameter measurement is shown in Figure 3. Images taken from SEM are first processed using Canny edge detector [11]. Figure 4 illustrates a sample image taken from SEM and its edges obtained by Canny edge detector. A normalized Radon transform is applied to the edge image to compute the projection of edges at different angles. Radon transform was earlier used in shape analysis studies [12]. Figure 4 shows the projections with respect 


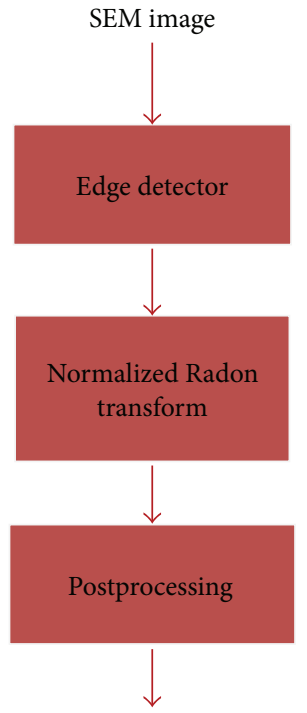

Fiber diameter

FIGURE 3: Flowchart of the proposed method.

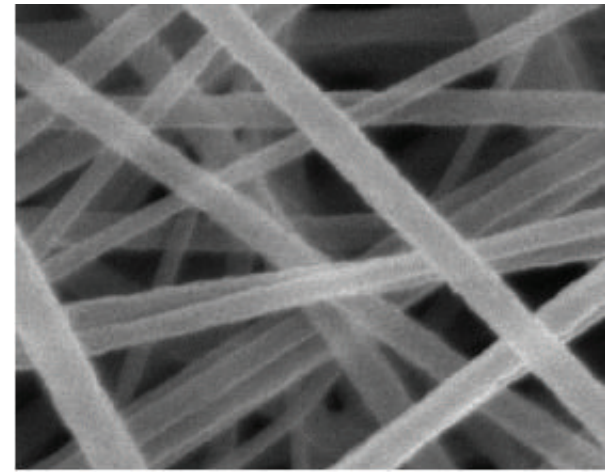

(a)

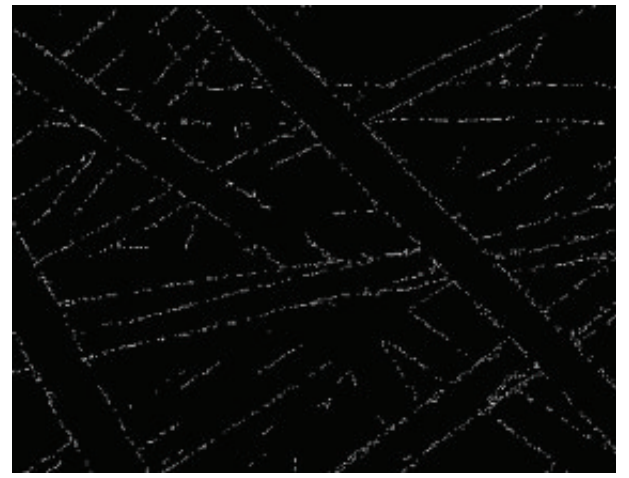

(b)

FIgURE 4: (a) Sample SEM image. (b) Edges of the sample image detected by Canny edge detector.

to the distance from the origin $(t)$ and the angle $(\theta)$. The mathematical expression for this projection is as follows:

$$
\delta(t-(x \cos \theta+y \sin \theta))
$$

where $\theta$ denotes the angle of the projection, $t$ denotes the distance of projection from the origin, and $\delta(\cdot)$ denotes the delta Dirac function [13]. In the proposed algorithm, the ratio of edges at different distance and angles to the distance of the corresponding projection within the image is computed as follows:

$$
R(t, \theta)=\frac{1}{L(t, \theta)} \sum_{x} \sum_{y} f(x, y) \delta(t-(x \cos \theta+y \sin \theta)),
$$

where $f(x, y)$ denotes the value of edge within the image at $(x, y)$ coordinate and $L(t, \theta)$ denotes the length of projection at distance $t$ and angle $\theta$ within the image (see Figure 5).

\section{Results}

In this study, 10 SEM images of the nanofibers produced using PAN/DMF, PU, PVA, PEO-PCL3 solutions of different concentrations, $12 \mathrm{kV}$ voltage, $14 \mathrm{~cm}$ needle to collector distance, and $1.2 \mathrm{~mL} / \mathrm{h}$ flow rate were used for automated nanofiber diameter measurement. 48 nanofibers were selected from these 10 SEM images. Diameters of the nanofibers were measured manually for the comparison at 568 locations.

Detected edges using Canny algorithm were searched with different distances and angles. A fiber edge that was found at $t=330$ and $\theta=46$ degrees is shown in Figure 6 . The normalized Radon transform (obtained from (2)) was computed which is shown in Figure 7. In this figure, the ratio values $(R(t, \theta))$ are shown on a logarithmic scale for a better visualization. Each node on this transform corresponds to a fiber in the SEM image. Some of these nodes are shown with arrows in Figure 7.

The ratio image is thresholded using a predetermined threshold value, 0.4 . Ratios that are over this threshold value 


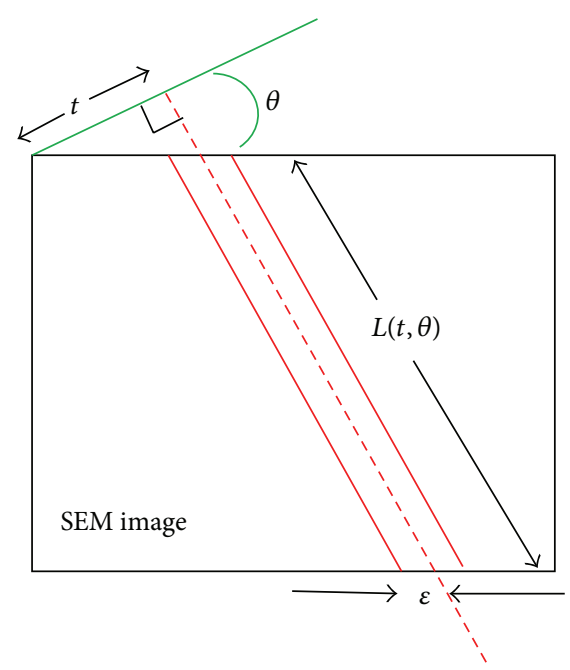

FIGURE 5: Normalized Radon transform, projection at distance $t$ and angle $\theta$ (denoted by the dashed line), distance of a projection within the image (denoted by $L$ ), and margins around the projection (denoted by $\varepsilon$ ).

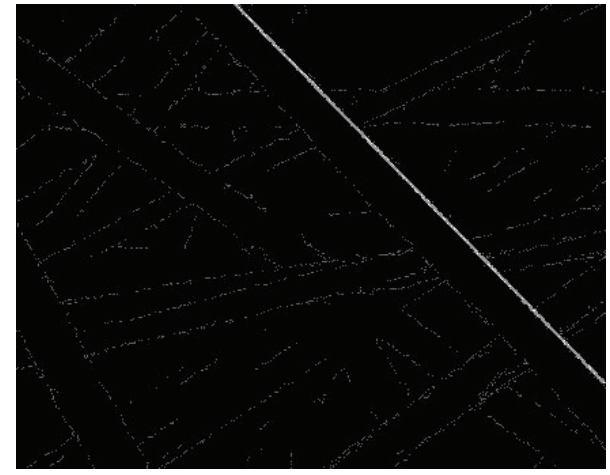

Figure 6: Projection at $t=330$ and $\theta=46$ overlaid on the edge image.

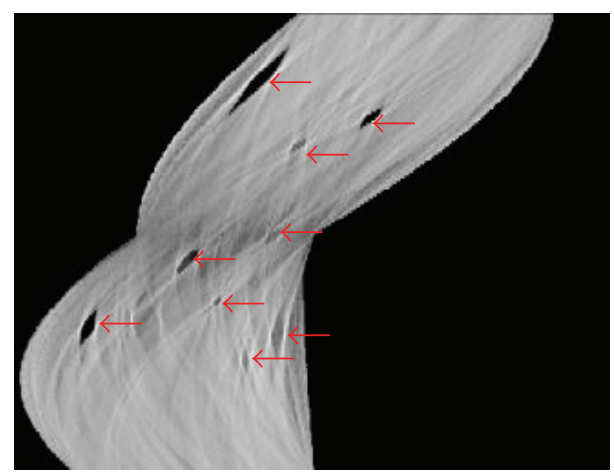

Figure 7: $R(t, \theta)$ matrix (logarithmic) is formed by projections taken at different distance and angles divided by the distance of the projection within the image.

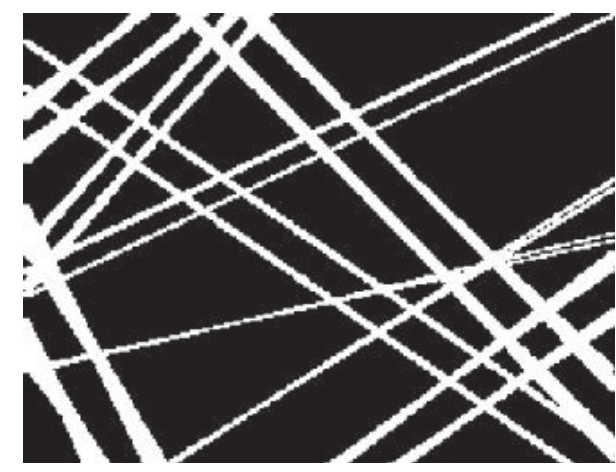

FIGURE 8: Thresholded and backprojected $R(t, \theta)$ image before postprocessing.

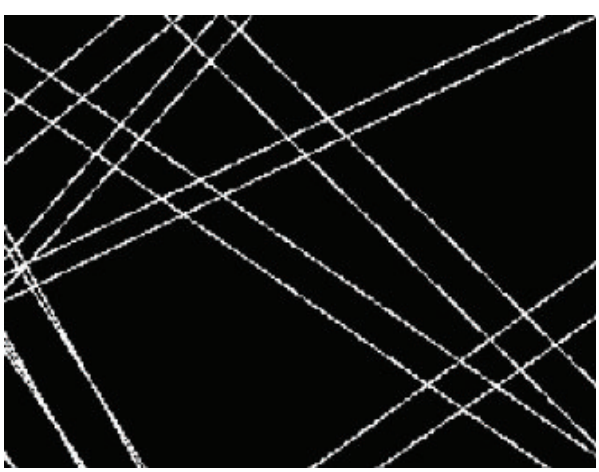

Figure 9: Thresholded and backprojected $R(t, \theta)$ image after postprocessing.

are backprojected to determine the edges of the fibers which are shown in Figure 8. There are many spurious edges for the same fiber. To remove the false edges, a postprocessing step is included. In this step, the edge corresponding to the highest ratio was accepted as the fiber edge and the rest were rejected. Furthermore, single edges were removed, and detected diameters that are out of the expected range for a fiber were also rejected in the postprocessing step.

The fibers corresponding to Figure 4(a) are shown in Figure 9. From this figure, it can be seen that 7 fibers are correctly located.

In Figure 10, some sample results from the proposed algorithm are shown. As it can be seen from these results, some of the fibers in the background could not be detected. The sensitivity of the algorithm is affected by the Canny algorithm parameters, the threshold value used for eliminating values of the ratio image below the threshold, and the margin size defined around each projection. The sensitivity may be increased by reducing the ratio threshold and increasing the margins around the projections. However, increasing sensitivity may result in more false edges in the SEM images. Therefore, there is a trade-off in determining the parameters of the algorithm.

In Figure 10(c), multiple edges found for the same fiber can be seen. Most of these artifacts were removed in the postprocessing step; however, some of them could not be yet filtered out due to the fixed threshold value and the margin size used. 


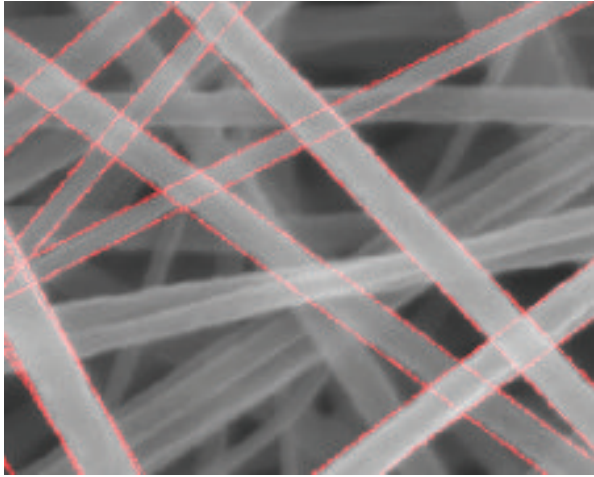

(a)

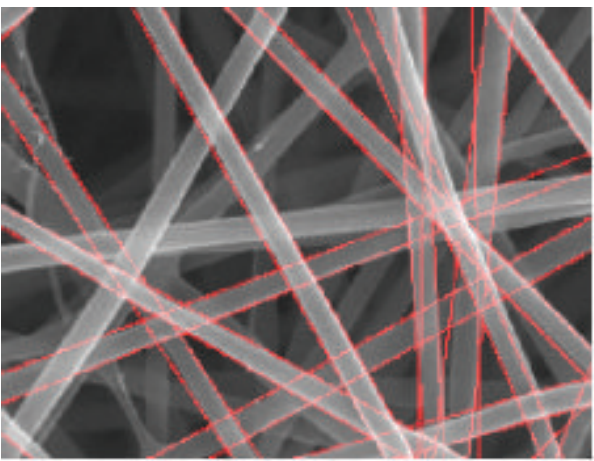

(c)

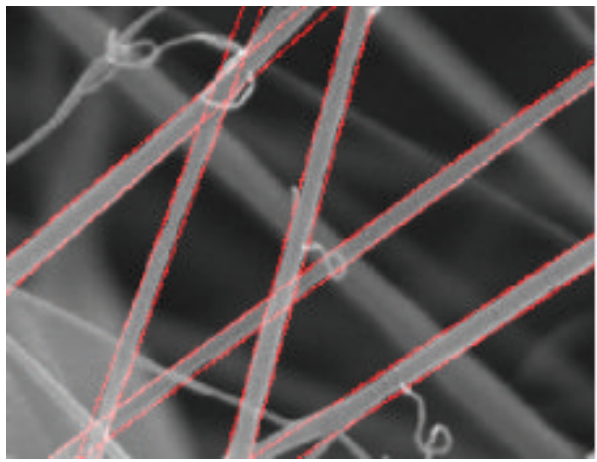

(e)

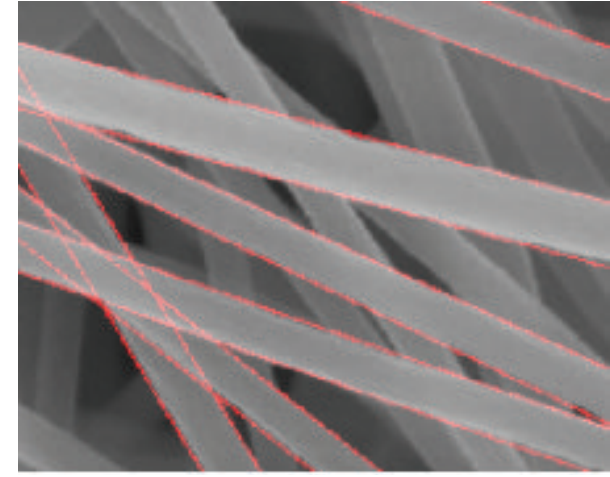

(b)

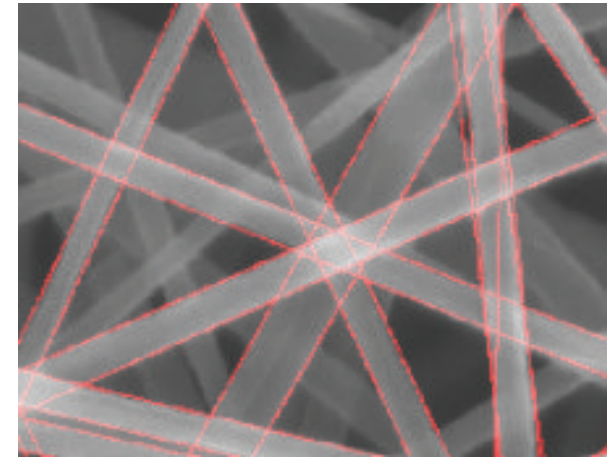

(d)

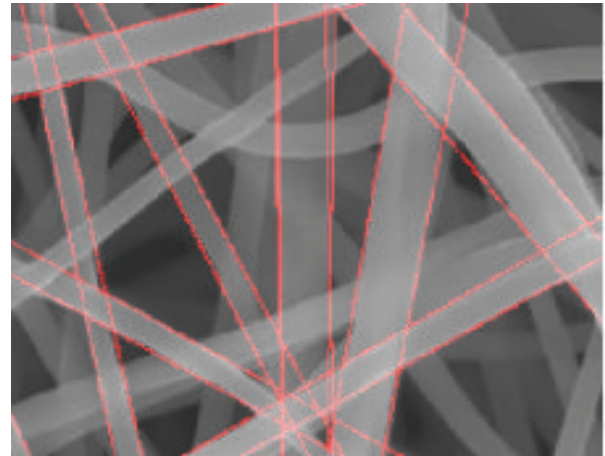

(f)

FIGURE 10: Sample results of the proposed algorithm for sample SEM images at different resolutions of PAN/DMF (8\% concentration) nanofiber mats.

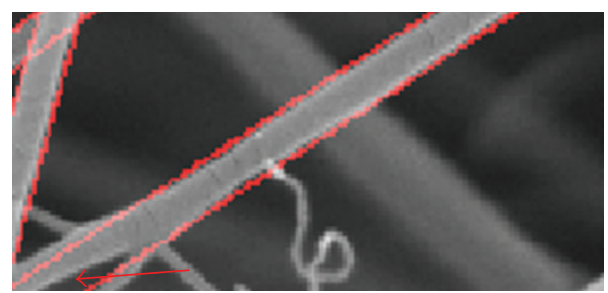

FIGURE 11: Proposed algorithm is robust to changes in the fibers such as bifurcations as shown in the lower left corner of the image.

In Figure 11, it is shown that the proposed algorithm is robust against bifurcations in the fibers. The average fiber diameter is our main interest, and the directional changes and bifurcations of fibers could not change the average fiber diameter computed by the proposed algorithm.

Manually measured fiber diameters at 568 locations and automatically measured fiber diameters by the proposed method were compared. The effectiveness of the proposed method was shown by obtaining very close values to the manually measured nanofiber diameter values as shown in Figure 12.

\section{Conclusion and Discussion}

In this study, an image analysis method for automated nanofiber diameter calculation is proposed. The algorithm 


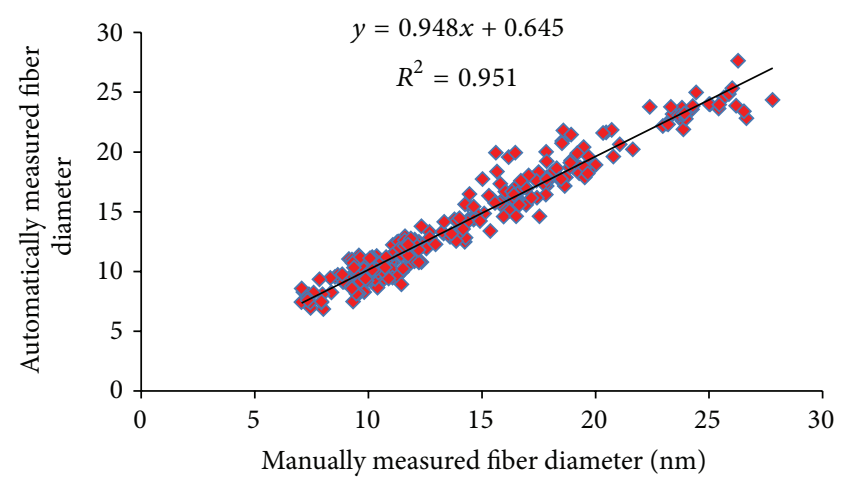

FIGURE 12: Comparison of manually measured fiber diameters and automatically measured fiber diameters in $\mathrm{nm}$ by the proposed method.

is tested on real SEM images. The results of the method are compared with the manually measured fiber diameters for 568 measurements. The results obtained with the proposed method are quite promising by generating results very close to the manual measurements $\left(R^{2}=0.956\right)$.

The proposed method has been shown to be robust to changes in the fibers such as bifurcations. The proposed method also addresses an important limitation of the former approaches that they first isolate each fiber in the image which is an error-prone process before measuring the diameter of each isolated fiber.

Currently, the proposed method's parameters are manually adjusted for different resolutions. In addition, false or multiple fiber edges may occasionally occur in the images. The sensitivity of the algorithm depends on threshold value and margin size. The lower threshold value and higher margin size are the more false edges. In the vice-versa case, there is a risk of not capturing some fibers in the images. The proposed algorithm can further be improved to catch more fibers within the image and filter out the false edges by selecting the parameters adaptive to the resolution of SEM images.

\section{Conflict of Interests}

The authors declare that there is no conflict of interests regarding the publication of this paper.

\section{Acknowledgment}

This research was supported by TUBITAK (Research Grant no. $108 \mathrm{M} 045)$. The authors would like to acknowledge and extend their heartfelt gratitude to the Scientific and Technological Research Council of Turkey (TUBITAK).

\section{References}

[1] B. Pourdeyhimi, R. Ramanathan, and R. Dent, "Measuring fiber orientation in nonwovens part I: simulation," Textile Research Journal, vol. 66, no. 11, pp. 713-722, 1996.
[2] B. Pourdehyhimi, R. Dent, A. Jerbi, S. Tanaka, and A. Deshpande, "Measuring fiber orientation in nonwovens part V: real webs," Textile Research Journal, vol. 69, no. 3, pp. 185-192, 1999.

[3] B. Pourdeyhimi and R. Dent, "Measuring fiber diameter distribution in nonwovens," Textile Research Journal, vol. 69, no. 4, pp. 233-236, 1999.

[4] M. Ziabari, V. Mottaghitalab, S. T. McGovern, and A. K. Haghi, "A new image analysis based method for measuring electrospun nanofiber diameter," Nanoscale Research Letters, vol. 2, no. 12, pp. 597-600, 2007.

[5] M. Ziabari, V. Mottaghitalab, and A. K. Haghi, "Distance transform algorithm for measuring nanofiber diameter," Korean Journal of Chemical Engineering, vol. 25, no. 4, pp. 905-918, 2008 .

[6] M. Ziabari, V. Mottaghitalab, and A. K. Haghi, "Application of direct tracking method for measuring electrospun nanofiber diameter," Brazilian Journal of Chemical Engineering, vol. 26, no. 1, pp. 53-62, 2009.

[7] A. H. Aydilek, S. H. Oguz, and T. B. Edil, "Digital image analysis to determine pore opening size distribution of nonwoven geotextiles," Journal of Computing in Civil Engineering, vol. 16, no. 4, pp. 280-290, 2002.

[8] R. Chhabra, "Nonwoven uniformity-measurements using image analysis," International Nonwoven Journal, vol. 12, no. 1, pp. 43-50, 2003.

[9] B. Xu and Y.-L. Ting, "Measuring structural characteristics of fiber segments in nonwoven fabrics," Textile Research Journal, vol. 65 , no. 1, pp. 41-48, 1995.

[10] T. Gumus, O. Erden, S. Gulsen, E. Oznergiz, and A. Demir, "A novel Multifunctional Electrospinning Platform (MEP)," in Proceedings of the Nanofibers for the 3rd Millennium, Raleigh, NC, USA, 2010.

[11] J. Canny, "A computational approach to edge detection," IEEE Transactions on Pattern Analysis and Machine Intelligence, vol. 8, no. 6, pp. 679-698, 1986.

[12] Z. Ma, B. Kang, K. Lv, and M. Zhao, "Nonlinear Radon transform using Zernike moment for shape analysis," Computational and Mathematical Methods in Medicine, vol. 2013, Article ID 208402, 9 pages, 2013.

[13] A. V. Oppenheim, A. S. Willsky, and S. Hamid, Signals and Systems, Prentice Hall, 1996. 

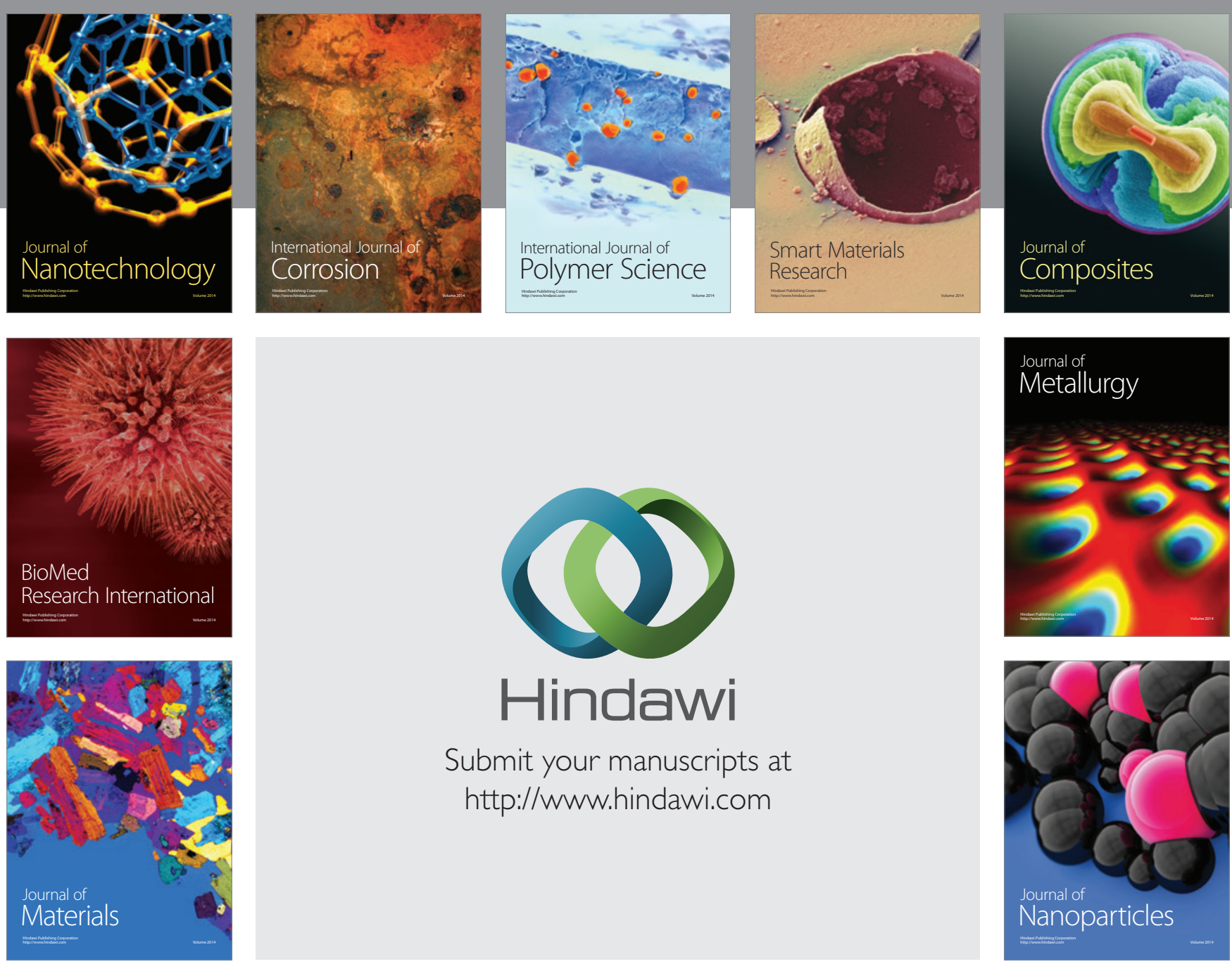

Submit your manuscripts at http://www.hindawi.com
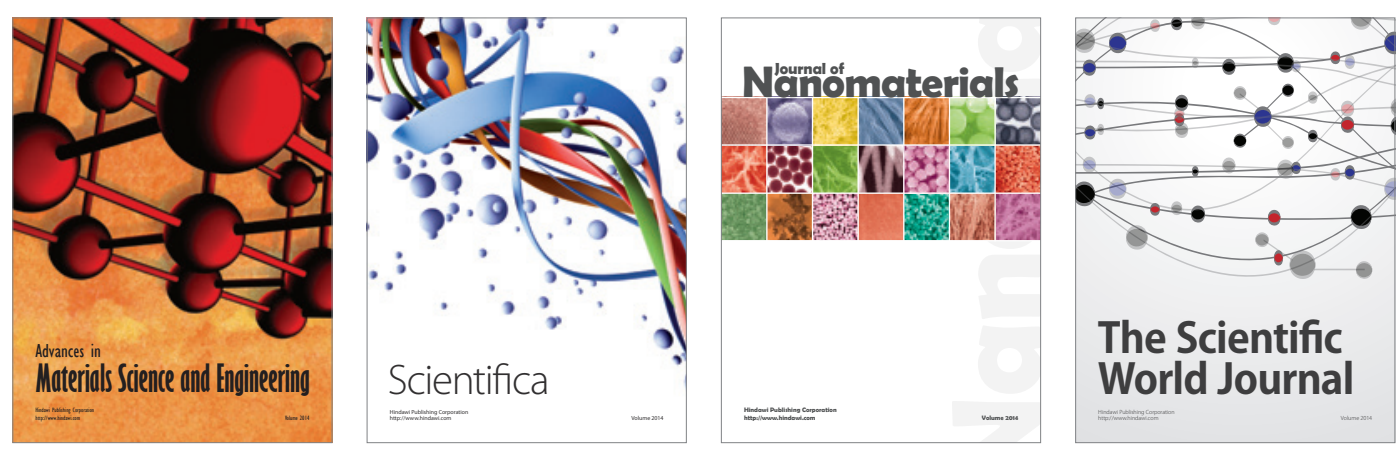

\section{The Scientific World Journal}
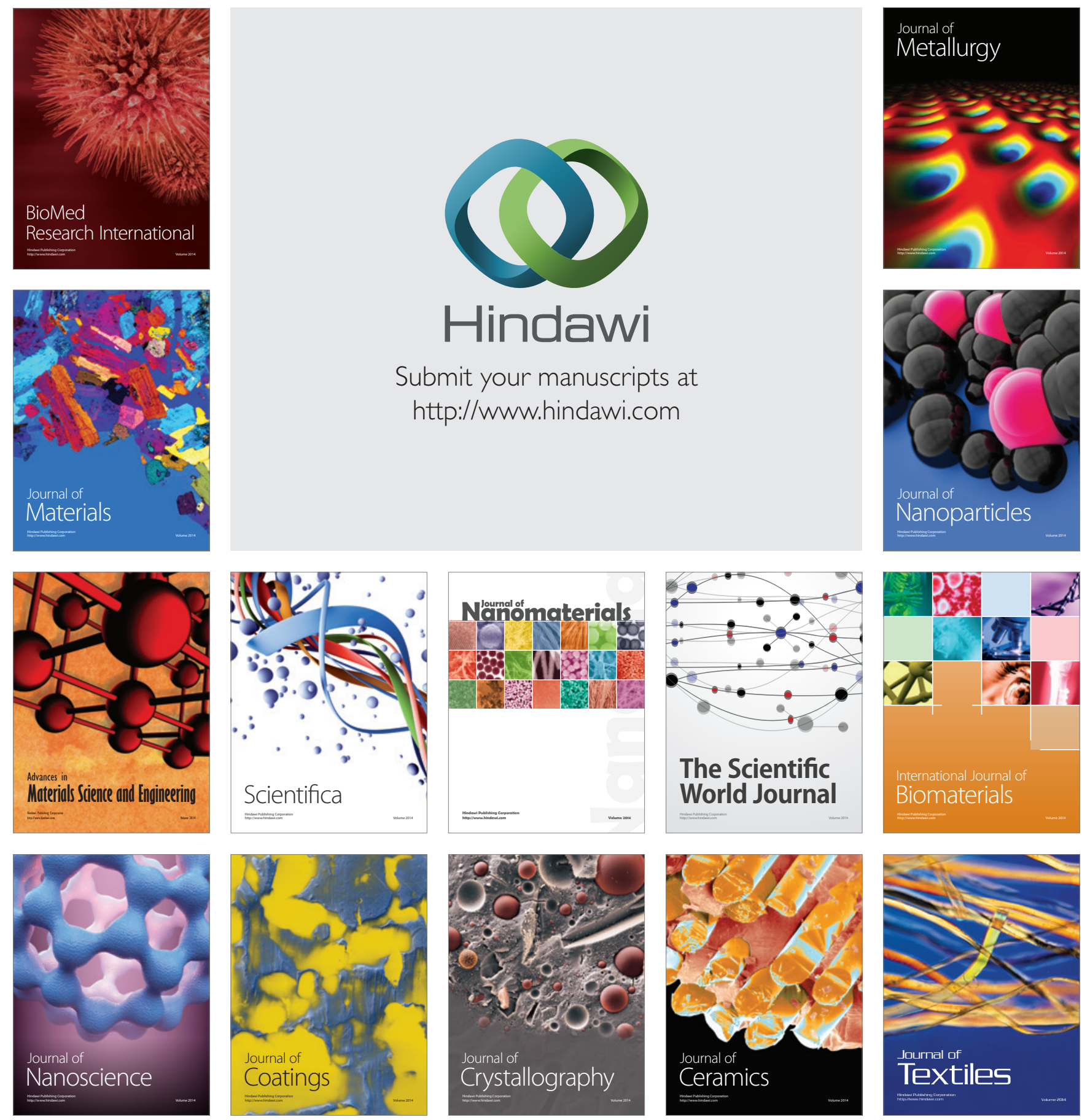